\title{
Extragalactic magnetic fields and directional correlations of ultra-high-energy cosmic rays with local galaxies and neutrinos
}

\section{Arjen van Vliet, ${ }^{a, *}$ Andrea Palladino, ${ }^{a}$ Walter Winter, ${ }^{a}$ Andrew Taylor $^{a}$ and Anna Franckowiak $^{a}$}

${ }^{a}$ DESY, Platanenallee 6, 15738 Zeuthen, Germany

E-mail: arjen.van.vliet@desy.de

Deflections of ultra-high-energy cosmic rays (UHECRs) in extragalactic magnetic fields (EGMFs) decrease the expected directional correlations between UHECR arrival directions on the one hand and UHECR source positions and neutrino arrival directions on the other hand. We use the recently observed correlation between UHECRs and local star-forming galaxies by the Pierre Auger Observatory to put limits on the EGMFs between these galaxies and the Milky Way [1]. In addition, using the same methods, we investigate whether correlations between UHECR and neutrino arrival directions can be expected [2]. We take into account deflections in extragalactic and Galactic magnetic fields, energy-loss interactions with background photon fields and UHECR spectrum and composition measurements. For a source density of star-forming galaxies, preliminary results indicate that strong EGMFs ( $B>0.6 \mathrm{nG}$ for a coherence length of $1 \mathrm{Mpc}$ at the $5 \sigma$ confidence level) are required to reproduce the level of anisotropy that Auger has observed. For more numerous sources, e. g. spiral galaxies, weaker EGMFs are allowed. However, this would suggest that UHECR acceleration occurs in many regular galaxies, which is rather difficult to motivate. We demonstrate that even for the weakest EGMFs the non-observation of neutrino multiplets strongly constrains the possibility to find neutrino-UHECR correlations. For star-forming galaxies, or more numerous sources, no neutrino multiplets or neutrino-UHECR correlations are currently expected.

$3^{\text {th }}$ International Cosmic Ray Conference (ICRC 2021)

July 12 th - 23rd, 2021

Online - Berlin, Germany

\footnotetext{
*Presenter
} 


\section{Introduction}

Recent advances in anisotropy analyses of the arrival directions of Ultra-High-Energy Cosmic Rays (UHECRs, cosmic rays with energies $E_{\mathrm{CR}} \gtrsim 1 \mathrm{EeV}$ ) provide indications for the possible origin of these messengers. The discovery of a dipole in the UHECR sky, directed away from the Galactic centre, suggests an extragalactic origin of these extremely energetic particles [3]. Excesses on intermediate angular scales indicate correlations with bright, nearby galaxies [4, 5]. In this case, the most significant correlation ( $4.5 \sigma$ post-trial significance) is found for a catalogue of local star-forming galaxies.

Besides UHECR anisotropies, the arrival directions of astrophysical neutrinos can also provide insight on cosmic-ray sources. The main method for creating neutrinos with energies $E_{v} \gtrsim 100 \mathrm{TeV}$ is by cosmic-ray interactions. Notably, correlations have been found between high-energy neutrino events and the blazar TXS 0506+056 [6] as well as the Tidal-Disruption Event (TDE) AT2019dsg [7]. The neutrinos associated with these sources are in the TeV-PeV energy range. Blazars and TDEs are, therefore, most-likely sites of PeV cosmic rays, but not necessarily for the acceleration of UHECRs. However, a common origin of PeV neutrinos and UHECRs could be established if correlations between UHECRs and neutrino arrival directions are found. The Pierre Auger Observatory (PAO), Telescope Array (TA) and IceCube are looking for such correlations, but no significant signals have been found yet [8].

UHECRs are charged particles, and are, therefore, deflected by the magnetic fields that they encounter when traversing the Universe. Both the Galactic Magnetic Field (GMF) and the Extragalactic Magnetic Fields (EGMFs) can deflect UHECRs. This influences the correlations that can be expected between source positions, or neutrino arrival directions, and UHECR arrival directions. However, the exact strength and directions of both the GMF and the EGMFs are currently not well known (see e.g. Refs. $[9,10]$ for overviews on Galactic and extragalactic magnetic fields).

Besides deflections in magnetic fields, another important factor contributing to whether correlations are observed is the local density of sources. A larger local source density means more sources contributing to the total flux and, therefore, a more isotropic sky. The absence of significant clustering in the arrival direction of UHECRs provides an indication for a minimal source density of $2 \cdot 10^{-5} \mathrm{Mpc}^{-3}$, under the assumption that the deflections from the sources are limited to within 30 degrees [11].

Neutrinos, on the other hand, do not get deflected in magnetic fields, but are hard to detect. The measured astrophysical neutrino flux extends to $\sim 6 \mathrm{PeV}$ [12], while below $\sim 200 \mathrm{TeV}$ it gets hard to distinguish astrophysical neutrino signals from the atmospheric background. The number of detected neutrinos with a low estimated background probability and good directional resolution is, therefore, rather limited. With 8 years of IceCube data, the highest-energy events of the throughgoing muon sample included 36 neutrino candidates [13]. In this through-going muon sample, no neutrino multiplets (two or more neutrinos coming from the same direction) have been discovered. This lack of neutrino multiplets can be used to constrain the local density of neutrino sources, as a low source density would mean that neutrino multiplets should have been observed. These constraints indicate that the local source density should be larger than $10^{-9}-10^{-5} \mathrm{Mpc}^{-3}$, depending on the source evolution with redshift and on whether steady or transient sources are considered (see e.g. Refs. $[14,15])$. 
In this work, based on Refs. [1,2], we investigate the possibility of finding correlations between neutrino and UHECR arrival directions. In addition, we study the implications of correlations between UHECR arrival directions and star-forming galaxies on the strength of local EGMFs. Besides the deflections in EGMFs, we take into account deflections in the GMF as well as the source density, interactions with background photon fields and UHECR spectrum and composition measurements by the PAO [16, 17].

\section{Method}

In short, we simulate UHECR sky maps for specific EGMF and GMF setups and local source densities $\rho_{0}$. For the correlations with star-forming galaxies, we determine which sky maps are viable considering the correlations found by the PAO. For the UHECR correlations with neutrinos, we determine in which sky maps significant correlations between UHECRs and neutrinos are found, under the most positive assumptions. How the UHECR and neutrino sky maps are created and analysed is shortly described in the following subsections, but for the full details see Refs. [1, 2].

\subsection{UHECR spectrum and composition}

For the UHECR spectrum and composition at the sources, we assume the parameters that gave the best fits to the measured spectrum and composition in Refs. [18, 19]. In Refs. [1, 2], we also show the results for pure proton scenarios as an indication for what would happen in a scenario with a much lighter composition.

For the correlations with neutrinos, we consider three different representative source evolutions with redshift $z$ : a negative $\left((1+z)^{-3}\right)$, a flat and a Star Formation Rate (SFR) [20] evolution. For each of these source-evolution scenarios the corresponding best-fit parameters for the UHECR spectrum and composition at the sources of Refs. $[18,19]$ are implemented. As energy range, we take $E_{\mathrm{CR}}>3 \mathrm{EeV}$, all UHECRs above the ankle, which can safely be assumed to be extragalactic. For the correlations with star-forming galaxies, the source evolution with redshift is not so important as the significant correlations are only with very local sources (distances $\lesssim 5 \mathrm{Mpc}$ ). Therefore, in this analysis, we only consider SFR source evolution, which is a good representative for star-forming galaxies. We adopt $E_{\mathrm{CR}}>38 \mathrm{EeV}$, the energy range for which the PAO found the most significant correlation with star-forming galaxies.

Starting from these source characteristics, we simulate the propagation of UHECRs from their sources to Earth in 1D using CRPropa 3 [21], including all relevant interactions with background photon fields. Here we assume a continuous distribution of identical sources. From these simulations, we obtain the distribution of UHECRs as a function of the redshift.

\subsection{Source catalogues and deflections in magnetic fields}

For the creation of each sky map, we create a different source catalogue. The sources in these catalogues are placed at random positions in the sky, at distances following the specific source evolution and the number of sources in the catalogues is determined by the local source density $\rho_{0}$. For the correlations with neutrinos, these sources produce both UHECRs and neutrinos. For the correlations with star-forming galaxies, the source catalogue is extended to also include NGC 253, NGC 4945, Circinus and M83 at their specific distances and positions in the sky (the minimal 
distance of the random sources is set to $6 \mathrm{Mpc}$ in this case). These four sources are the sources that dominate the star-forming galaxy correlations found by the PAO.

We also use CRPropa 3 to simulate the expected deflections of UHECRs in EGMFs from their specific sources. For the correlations with neutrinos, we do this using the weakest EGMF model of Ref. [22]. For that paper, the authors created six different EGMF models which all resemble the local Universe, but had different assumption for the seeding of the EGMFs. By choosing the weakest of these models, we obtain a rather conservative estimate for the deflections of UHECRs in EGMFs, meaning we test the most optimal case for UHECR-neutrino correlations. From these simulations, we obtain a distribution of deflections for each redshift bin.

In the scenario with correlations with star-forming galaxies, we only consider deflections from the four sources that dominate the signal. All other sources in the source catalogues are placed at random positions in the sky and only contribute to an isotropic background. As EGMF setup, in this case, we use random turbulent fields with a Kolmogorov spectrum with RMS magnetic-field strengths $B_{\mathrm{RMS}}$ and maximum correlation lengths $l_{\text {coh }}$. We scan over $\tilde{B}=B_{\mathrm{RMS}} \times \sqrt{\ell_{\mathrm{coh}}}$. The goal, in this case, is to determine for which EGMF strengths between the dominant sources and the Milky Way the anisotropy detected by the PAO can be reproduced. As these sources are all very nearby (distances $\lesssim 5 \mathrm{Mpc}$ ), this analysis is only sensitive to local EGMFs, which are likely produced by magnetised haloes around local galaxies. These local EGMFs are well represented by random turbulent fields as the distances are too small to expect additional structure from galaxy clusters, filaments or sheets. The chosen range of $\tilde{B}$ represents Larmor radii between $\sim 0.5$ and $\sim 50 \mathrm{Mpc}$ for typical UHECR rigidities, and, therefore, covers the range from small to large deflections for typical source distances of $\sim 5 \mathrm{Mpc}$. With these EGMF setups, probability density sky maps are created by simulating the trajectories of UHECRs from the four dominant sources to Earth, taking into account all relevant energy-loss processes.

As typical GMF strengths and correlation lengths are rather uncertain, we show the results both with and without deflections in the GMF. While the scenarios with GMF deflections should be considered more realistic, the scenarios without GMF deflections can be considered as extreme cases with limited deflections. As GMF model, we use the JF12 model from Refs. [23, 24]. For the UHECR-neutrino correlations scenario, we use the parameterisation of the distribution of deflections as a function of the rigidity of the UHECRs as given in Ref. [25]. We obtain the distribution of rigidities from the 1D simulations. For the UHECR correlations with star-forming galaxies, we run the GMF lensing method of CRPropa 3 on the probability density maps obtained after EGMF deflections. In this way, we produce probability density maps for the four dominant sources after deflections in the GMF.

\subsection{Sky maps and the excess over the background}

With these source catalogues and UHECR distance and deflection distributions, we create sky maps for specific values of $\rho_{0}$ and magnetic-field setups. Each source in the source catalogues is assigned a chance for producing an observed UHECR based on the distance distribution of UHECRs, and the sources that produce observed UHECRs are determined randomly based on these chances. The actual position in the sky of these UHECRs is then determined by taking into account the deflections in the specific magnetic-field setup. 
In the UHECR-neutrino correlations scenario, the amount of deflection is randomly determined following the corresponding deflection distribution, first for extragalactic deflections and secondly for Galactic deflections (if applied). The direction of the deflection is chosen randomly on the sphere of the sky with equal probabilities for all directions. For the significance analysis it does not matter in which direction the deflection was, as only the total number of UHECRs within a circle around neutrino arrival directions are considered. Besides UHECRs, neutrino arrival directions are added to these sky maps as well. The neutrino positions are drawn randomly from the same source catalogue (without any deflections), but in this case the probabilities assigned to the sources follow the neutrino distribution with redshift (taking into account adiabatic energy losses only). In this way, sky maps are created with 36 neutrinos (the number of highest-energy events in the through-going muon sample with 8 years of IceCube data [13]) and 135k UHECRs (about the numbers of UHECRs with $E_{\mathrm{CR}}>3 \mathrm{EeV}$ detected by the PAO and TA combined).

For the star-forming galaxies case, the arrival directions of UHECRs from the four dominant sources are randomly drawn from the probability density maps corresponding to the specific magnetic-field setup. The arrival directions of UHECRs from the background sources are placed at random position on the sky map assuming an isotropic distribution. The exposure of the PAO is taken into account in the creation of both these probability density maps. In this way, sky maps are created with 894 UHECRs (the number of detected UHECRs with $E_{\mathrm{CR}}>39 \mathrm{EeV}$ used in the analysis of the PAO [4]).

For each combination of magnetic-field setup and $\rho_{0}, 1000$ sky maps are created from different source-catalogue realisations. For each of the obtained sky maps, the excess of UHECRs over an isotropic background is determined in angular windows around either neutrino arrival positions or the positions in the sky of the four dominant star-forming galaxies. The radii of these angular windows is scanned over to determine the largest excess.

For the scenario with neutrino correlations, we evaluate, for each sky map, the significance of the UHECR-neutrino correlations using a Poissonian likelihood $\chi^{2}$ analysis. We then determine for which local densities (with or without GMF deflecions, for transient and steady sources and for the 3 source evolution scenarios separately) the median $5 \sigma$ discovery potential is reached. In addition, we compute the probability to observer neutrino multiplets for all local source densities.

In the case of the correlations with star-forming galaxies, the resulting optimal angular window $\theta_{\mathrm{XS}}^{\mathrm{SFGs}}$ and fraction of UHECRs associated to the four dominant sources $f_{\mathrm{XS}}^{\mathrm{SFGs}}$ are compared with the results of the correlation analysis of the PAO. In this way, we determine, for all combinations of $\tilde{B}$ and $\rho_{0}$, the compatibility with the PAO results expressed in number of $\sigma$.

\section{Results}

The results for the UHECR-neutrino correlations are shown in Fig. 1, for the 3 different source evolution scenarios and steady and transient sources separately. For all scenarios, UHECR-neutrino correlations are only expected if they are produced by relatively rare sources $\left(\rho_{0} \lesssim 10^{2} \mathrm{Gpc}^{-3}\right)$. Keep in mind that we are testing an optimal case for UHECR-neutrino correlations, e.g. by assuming that all UHECRs and neutrinos are produced in the same sources and by implementing a weak EGMF setup. Even in this optimal case, the absence of neutrino multiplets constrains the whole parameter space where UHECR-neutrino correlations are expected, if deflections in both the EGMF and GMF 

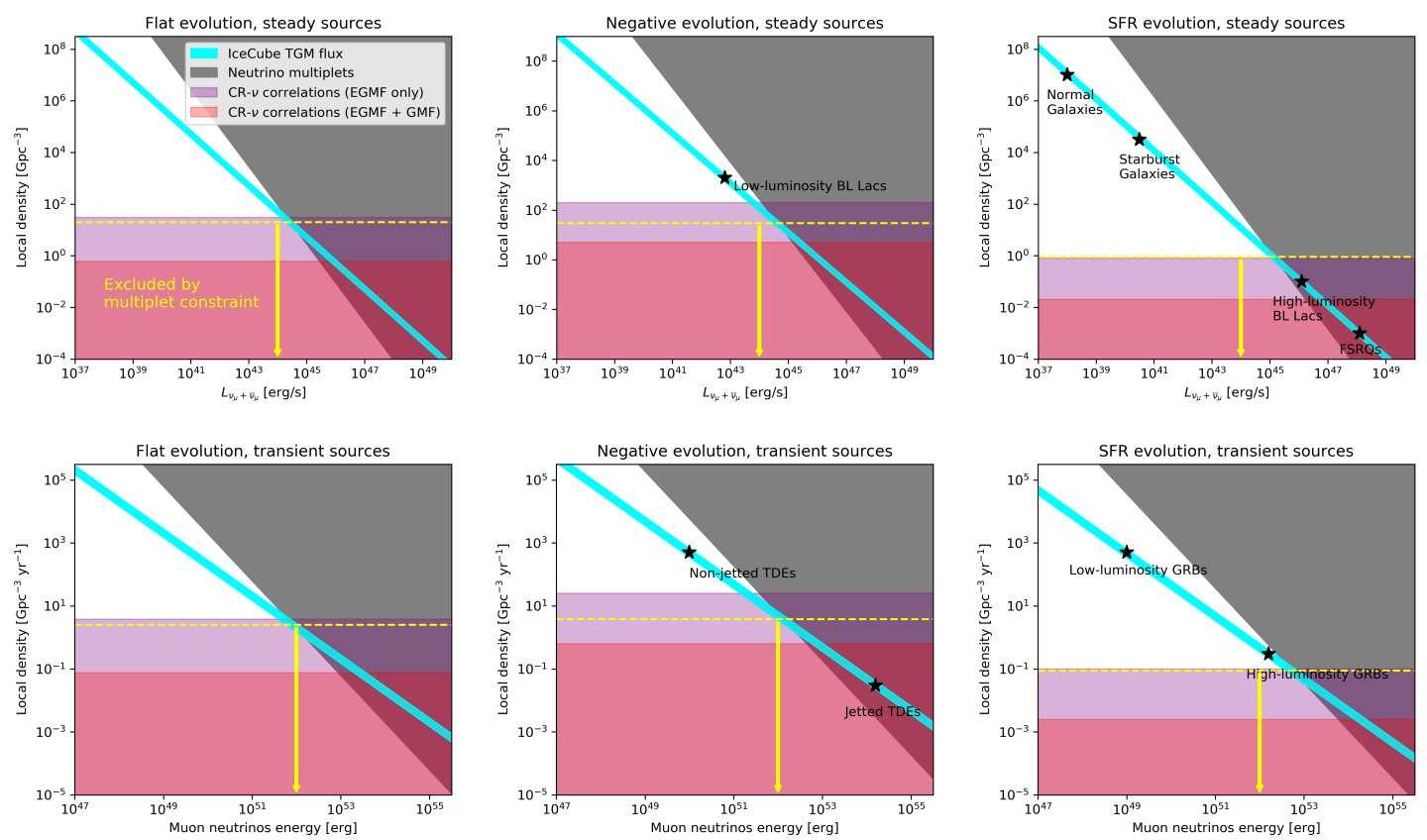

Figure 1: Through-going muon flux levels (cyan), parameter spaces with $\geq 90 \%$ chance of observing neutrino multiplets (gray) and local-density ranges with at least a median $5 \sigma$ discovery potential for UHECR-neutrino correlations including (red) and excluding (purple) deflections in the GMF. The yellow lines show the intersect points of the neutrino flux with the neutrino multiplet region, indicating that local source densities below these lines are excluded by the multiplet constraints. The results are shown for flat (left), negative (middle) and SFR (right) source evolutions, as well as for steady (top) and transient (bottom) sources. The source densities and source evolutions of typical steady or transient source candidates are indicated by stars. The figures are obtained from Ref. [2].

are considered (the most realistic scenario). UHECR-neutrino correlations are only likely if the sources follow a negative evolution with redshift and if deflections of UHECRs in the GMF are smaller than predicted by the JF12 GMF model.

The preliminary results for the UHECR correlations with star-forming galaxies are shown in Fig. 2, for both with and without deflections in the GMF. Considering that this analysis is based on the correlations that the PAO found with local star-forming galaxies, it is natural to assume that the isotropic background is produced by the same source class. In that case, for a local density of star-forming galaxies, preliminary $5 \sigma$ lower limits for 1 degree of freedom on $\tilde{B}$ are obtained of $\tilde{B}>0.64 \mathrm{nG} \mathrm{Mpc}^{1 / 2}$, from the scenario with deflections in the GMF.

For the full range of $\rho_{0}$ that we consider, an anti-correlation is visible between $\rho_{o}$ and $\tilde{B}$. This is expected, as both larger deflections in magnetic fields and a larger number of sources can reduce any anisotropies in the sky. Too strong magnetic fields, or too large source densities, give a high level of isotropy incompatible with the correlations found by the PAO. We, therefore, obtain $90 \%$ confidence level upper bounds on $\tilde{B}$ and $\rho_{0}$ of $\tilde{B}<24 \mathrm{nG} \mathrm{Mpc}^{1 / 2}$ and $\rho_{0}<0.074 \mathrm{Mpc}^{-3}$, from the scenario without deflections in the GMF. 

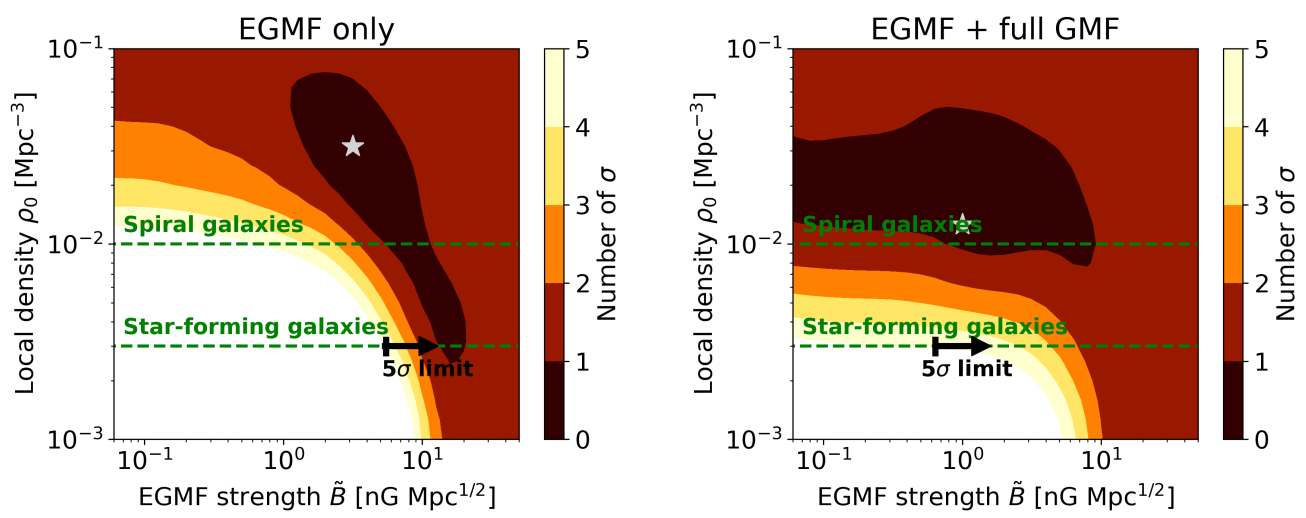

Figure 2: Preliminary results for the allowed region of EGMF strength and local source density compared with the PAO correlation analysis for star-forming galaxies of Refs. [4,5]. The number of sigma is obtained using the $\chi^{2}$ for two degrees of freedom. Two different scenarios are shown, with (right) and without (left) deflections in the GMF. The best-fit points are indicated by stars and the source density of spiral galaxies and of star-forming galaxies [26] are indicated by horizontal lines. The $5 \sigma$ lower limits on $\tilde{B}$ for the source density corresponding to star-forming galaxies are shown as well. The figures are obtained from Ref. [1].

\section{Conclusions}

We have investigated the effects of deflections in EGMFs on correlations between the arrival directions of UHECRs on the one hand, and the arrival directions of neutrinos or the positions in the sky of nearby star-forming galaxies on the other hand. Besides deflections in EGMFs, we take into account deflections in the GMF, the local source density, energy-loss interactions during propagation and spectrum and composition measurements by the PAO.

Our results show that, given the neutrino multiplet constraints, it is unlikely that any significant UHECR-neutrino correlations will be found soon. The strongest potential for finding UHECRneutrino correlations is when the common sources of UHECRs and neutrinos follow a negative source evolution. But even in that case, neutrino-UHECR correlations are only expected under our optimal assumptions if the UHECR deflections are less than predicted by the JF12 GMF model, or if IceCube observes a neutrino multiplet in the high-energy through-going muon sample in the very near future.

On the other hand, if significant neutrino-UHECR correlations are found in the near future nonetheless, this would give a strong indication for a local source density in the $10-10^{3} \mathrm{Gpc}^{-3}$ range, for a negative redshift evolution of the sources and for a common origin of UHECRs and high-energy neutrinos.

Our preliminary results on the correlations between UHECR arrival directions and local starforming galaxies indicate that either relatively strong local EGMFs are required $(B>0.64 \mathrm{nG}$ for correlation lengths of $1 \mathrm{Mpc}$ ), or that UHECRs are accelerated at very many sources (source densities close to that of spiral galaxies). It is, however, rather hard to motivate from the current knowledge of UHECR acceleration that UHECRs are produced in such abundant sources.

Acknowledgements This project has received funding from the European Research Council (ERC) under the European Union's Horizon 2020 research and innovation program (Grant No. 
646623).

\section{References}

[1] A. van Vliet, A. Palladino, A. Taylor and W. Winter, arXiv:2104.05732, submitted to MNRAS.

[2] A. Palladino, A. van Vliet, W. Winter and A. Franckowiak, MNRAS 494 (2020) 4255.

[3] Pierre Auger Collaboration, Science 357 (2017) 1266.

[4] Pierre Auger Collaboration, ApJ 853 (2018) L29.

[5] L. Caccianiga for the Pierre Auger Collaboration, PoS ICRC2019 206.

[6] M. G. Aartsen et al., Science 361 (2018) eaat1378.

[7] R. Stein et al., Nature Astron. 5 (2021) 510.

[8] IceCube, Pierre Auger and Telescope Array Collaborations, JCAP 01 (2016) 037.

[9] M. Haverkorn, Astrophys. Space Sc. L. 407 (2015) 483.

[10] R. Durrer and A. Neronov, Astron. Astrophys. Rev. 21 (2013) 62.

[11] Pierre Auger Collaboration, JCAP 05 (2013) 009.

[12] IceCube Collaboration, Nature 591 (2021) 220.

[13] C. Haack for the IceCube Collaboration, PoS ICRC2017 1005.

[14] IceCube Collaboration, Eur. Phys. J. C 79 (2019) 234.

[15] IceCube Collaboration, PRL 122 (2019) 051102.

[16] I. Valiño for the Pierre Auger Collaboration, PoS ICRC2015 271.

[17] Pierre Auger Collaboration, PRD 90 (2014) 122005.

[18] Pierre Auger Collaboration, JCAP 04 (2017) 038.

[19] R. Alves Batista et al., JCAP 01 (2019) 002.

[20] H. Yüksel et al., ApJ 683 (2008) L5.

[21] R. Alves Batista et al., JCAP 05 (2016) 038.

[22] S. Hackstein et al., MNRAS 475 (2018) 2519.

[23] R. Jansson and G. R. Farrar, ApJ 757 (2012) 14.

[24] R. Jansson and G. R. Farrar, ApJ 761 (2012) L11.

[25] G. R. Farrar and M. S. Sutherland, JCAP 05 (2019) 004.

[26] C. Gruppioni et al., MNRAS 432 (2013) 23. 